\title{
CAPACIDAD INSTITUCIONAL: REVISIÓN DEL CONCEPTO Y EJES DE ANÁLISIS
}

\author{
Angélica Rosas ${ }^{(\cdot)}$ \\ Universidad Autónoma Metropolitana \\ (Xochimilco, México)
}

\begin{abstract}
RESUMEN
Muchos estudios que evalúan políticas concluyen que los fracasos de implementación se deben a la falta de capacidades estatales. Sin embargo ¿a qué se refiere cuando se habla de capacidad institucional? ¿Cuáles son sus diversas concepciones? ¿y cuáles los ejes para su análisis? ¿qué indicadores existen para su evaluación? Se busca realizar un aporte a la conceptualización de las capacidades institucionales, y presentar una metodología para analizarla. Una de las reflexiones es que la capacidad institucional ha evolucionado para responder a nuevos problemas y sistemas de gestión de las agencias estatales, sin embargo, el concepto ha sido utilizado arbitrariamente asumiendo una diversidad de ejes de análisis e indicadores que ha dificultado contar con esquemas para su evaluación.
\end{abstract}

PALABRAS CLAVE:

capacidad institucional, capacidad administrativa, capacidad política, estado.

(•)E-mail: anrosas@gmail.com

\section{ABSTRACT}

Many studies that evaluate public policy conclude that most of the failures are due to a lack of institutional capacities of state agencies. However, what do we mean by institutional capacity? Which are their different conceptions? And what are the axes for its analysis? This work seeks to contribute to the conceptualization of institutional capacity and it presents a methodology that allows its analysis. One of the conclusions is that institutional capacity has evolved to give answer to new problems and new public management systems however the concept was used arbitrarily and the wide range of views and variables used have undermined effective evaluations.

\section{KEY WORDS:}

institutional capacity, administrative capacity, political capacity, state. 


\section{INTRODUCCIÓN}

Nuestras sociedades actuales y sus gobiernos enfrentan problemas que se han atendido desde hace varios lustros; la pobreza, el desempleo, el déficit en la salud pública, la poca calidad de la educación, son ejemplo de ello. Algunos otros se han presentado en décadas recientes, es el caso del terrorismo, la inseguridad, el impacto y la vulnerabilidad ante el cambio climático, la disposición de residuos sólidos, la expansión de la mancha urbana en suelo de conservación, y sobrexplotación de los mantos acuíferos. Todos ellos son problemas que no se han podido resolver y en algunos casos se han dejado sentir con mayor fuerza y han impactado cada vez más a un mayor número de poblaciones.

Actualmente se cuenta con literatura que da evidencia de que para la elección de los objetivos de gobierno, para la selección de los asuntos públicos, el tipo y forma de tratamiento y sus alcances dependen, mayormente, de las capacidades institucionales reales con las que cuentan las organizaciones gubernamentales (Rosas, 2015a; Alonso et al, 2015; Palermo, 2008; y Completa, 2016). Principalmente el problema de la eficacia en la atención de los asuntos públicos tiene que ver con las capacidades del gobierno para hacer efectiva sus decisiones. 
Aunado a ello somos testigos de procesos de modernización del Estado implementados en América Latina que vienen acompañados de nuevos roles y nuevos estilos de gestión para los gobiernos, principalmente los locales que demandan de las dependencias estatales una mayor capacidad para producir resultados en cuanto a la atención de los problemas públicos, mejores niveles de especialización y capacitación de sus funcionarios, una mejor cooperación y coordinación entre las organizaciones, una mayor participación de los actores que se encuentran en la sociedad, entre otros.

Ante la permanencia y complejidad creciente de los asuntos públicos, la falta de eficacia de las políticas públicas y la demanda emergente para cambiar el estilo de la gobernación, los gobiernos y los encargados de elaborar políticas (policy-making) se encuentran ante el reto de realizar esfuerzos continuos para mejorar sus intervenciones y obtener resultados efectivos. De ahí la necesidad de conocer y asumir su capacidad institucional; así como elevarla, mejorarla, reconstruirla o fortalecerla para el manejo eficaz de la "cosa pública". Ahora bien ¿a qué se refiere cuando se habla de capacidad institucional? ¿Qué implicaciones tienen las diversas concepciones de capacidad institucional para su análisis? ¿Cuáles son los ejes primordiales de su análisis?

Este trabajo busca contribuir a superar tres cuestiones que afectan los estudios de capacidad institucional. Primero, dicho concepto ha sido utilizado de manera arbitraría por diferentes autores, lo cual ha hecho que sea casi imposible diferenciarlo de otros conceptos, por ejemplo, con «desempeño gubernamental», "desarrollo institucional» o "buen gobierno". También ha sido analizado asumiendo diferentes ejes de análisis e indicadores, lo que ha dificultado contar con esquemas integrales y válidos para su evaluación. Por último, y no por ello menos importante, la capacidad institucional se ha analizado y evaluado sin asumir, clarificar y validar un método, pensando éste no como la técnica, es decir, como los instrumentos o procedimientos que nos ayudan a recabar, reunir, manipular y ordenar la información sobre la capacidad institucional, sino como un asunto de concepción, como el acto mismo de pensarlo.

En términos conceptuales, la capacidad institucional ha tenido una evolución y tratamiento distinto, por ello, en un primer momento, se presenta el desarrollo y definiciones que ha tenido el concepto. Posteriormente se reflexiona en torno a la importancia que tiene la capacidad institucional para la atención eficaz y eficiente de los problemas públicos Por último, se presentan ejes primordiales de análisis y una propuesta analítica que permiten demostrar la presencia de capacidad institucional. El artículo culmina con algunas reflexiones finales. 
La estrategia metodológica consistió en la revisión de bibliografía especializada con el fin de identificar los planteamientos teóricos, los ejes primordiales de su análisis y las formas que se han propuesto para analizar la capacidad institucional. No es el interés del presente trabajo realizar una revisión exhaustiva de la literatura sobre el tema sino de identificar los principales estudios que han contribuido con la evolución del concepto, su resignificación y tratamiento.

\section{EVOLUCIÓN DEL CONCEPTO}

El término capacidad institucional ha evolucionado y se ha resignificado de acuerdo al contexto histórico-temporal. Como tal no sólo hay un cambio en la formación del concepto en sentido estricto y propio, sino también en su tratamiento y sistematización.

El interés por la capacidad institucional empieza a manifestarse en las agendas académicas y políticas, justo cuando se exponen los límites del fundamentalismo del mercado, el fracaso de las políticas del «Estado Mínimo» en América Latina y se comienza con la aplicación de las reformas de segunda generación del Estado, centradas en promover un conjunto de medidas administrativas al interior del mismo para lograr una eficiencia, efectividad, y calidad del servicio. Se buscaba cambiar la visión de "menos Estado" a «mejor Estado", logrando con ello poner de nuevo al Estado en un primer plano mediante el fortalecimiento y mejoramiento de la capacidad de gestión de sus organismos y entidades, capacidades que permitan formular, implementar, coordinar, monitorear, evaluar y rendir cuenta, en el marco de un sistema de gestión pública (Oszlak, 2004). A partir de este momento se identifican al menos tres momentos de cambio.

Estudios dan evidencia que durante la primera mitad de la década de los ochenta existía "una preocupación [...] del Estado por la construcción y/o reconstrucción de las instancias habilitadas para gobernar procesos complejos, tras recuperar cierto terreno con relación a las posturas en boga hasta años atrás cuando se proponía la extrema reducción del mismo, con la promesa de que el mercado generaría por sí sólo las condiciones de crecimiento e incluso integración social» (Repetto, 2003: 1). Es en este contexto que, como señala Proenza (2014) "se precisaba "adecuar las instituciones" a las nuevas condiciones histórico concretas por las que estaba transitando" (p. 88).

Es así que se manifestó la necesidad de mejorar las habilidades para cumplir las funciones del Estado mediante el fortalecimiento institucional a través de la reingeniería organizacional (Ospina, 2002). Esta forma de conceptualizarlo 
buscaba mejorar la actuación estatal a través del mejoramiento de la estructura interna, los sistemas y las estrategias de la organización. El análisis se centró en identificar cómo el Estado podría lograr que las políticas fueran eficientes y eficaces; sin embargo la atención se limitó al aparato organizacional responsable de las políticas públicas, a aspectos técnico-administrativos que impedían el éxito de las políticas, en particular en la construcción, mejoramiento y fortalecimiento de la estructura interna, sistemas, estrategias de la organización y en los procedimientos; y asumiendo un alejamiento y aislamiento de sus cuadros técnico-burocráticos de los grupos de interés, su entorno y sociedad. No se reconocía que la eficiencia y eficacia de las políticas también podría estar determinada también por la relación que se pudiera establecer entre las dependencias estatales y la sociedad.

Es así como las intervenciones buscaban solucionar problemas concretos de la organización, es el caso del reclutamiento con base en el mérito, la promoción y el ascenso, los salarios y el escalafón (Sikkink, 1993; y Evans, 1996). Con esta noción se reconoce que el éxito o fracaso de cualquier acción o política se puede deber a la capacidad interna con la que contaba el Estado para asumir y cumplir sus funciones y responsabilidades.

En los noventa se reconfigura el término a construcción de capacidad institucional, esto debido a que en esta época se reconoce que ni los mercados ni las democracias podrían funcionar bien a menos que los gobiernos fueran capaces de diseñar e implementar políticas públicas apropiadas, administrar los recursos equitativamente, con más transparencia y eficiencia, y responder efectivamente a las demandas ciudadanas para el bienestar social. La nueva aspiración en el cambio se convierte entonces en el "buen gobierno" cuya característica fundamental era la construcción de capacidades institucionales como base del proceso (Grindle, 1997b; y Ospina, 2002).

El concepto de "construcción de capacidad institucional" asume una visión más compleja y un enfoque de sistemas que ubica los problemas organizacionales dentro de un entorno con varios niveles, actores e influencias, y con importantes interdependencias entre éstos (Ospina, 2002; y UNICEF, 1999). Actores públicos, privados, y sociales que deben participar en los procesos de identificación y resolución de los problemas públicos con miras al logro de objetivos de desarrollo (Banco Mundial, 1997).

Bajo esta visión se presentan posturas que reducen la utilidad del concepto, ya que borran las fronteras que distinguen las intervenciones para construir capacidad institucional de las intervenciones para generar desarrollo sostenible. Son definiciones que proporcionan una visión muy amplia que equipara erróneamente capacidad con desarrollo. ${ }^{1}$ 
Bajo la misma noción de "construcción de capacidad institucional» se desarrollan posturas que son catalogadas como intermedias entre una visión demasiado amplia que equipara "capacidad" con "desarrollo" y una visión demasiado reducida que la iguala con el desarrollo de habilidades y competencias en el recurso humano. Es el caso de Grindle con su discusión sobre la construcción de capacidad sostenible en el sector público con base al desarrollo humano, el fortalecimiento organizacional y la reforma institucional (Grindle, 1997a).

En la primera década del siglo XXI vuelve a experimentar una variación, ahora a capacidad institucional, la cual se reconfigura en el marco de la gobernanza — governance-. Es así como la concepción de capacidad hace referencia a la participación de los grupos sociales, el entorno y la ciudadanía en las decisiones que se toman en la gestión pública, en particular en el proceso de la política pública, desde su formulación y diseño hasta su implementación y evaluación, con el fin de fortalecer la organización gubernamental y su actuación a través de su diversidad, pluralismo, autonomía e interdependencia con relación a otras organizaciones en el sistema (Savitch, 1998); así como también promoviendo y creando redes de interacción entre las organizaciones del sector público, privado y las organizaciones no gubernamentales (Savitch, 1998 y Hall, 2002).

Este nuevo término plantea una concepción que va más allá del aparato administrativo estatal, ahora alude a la buena gestión de los asuntos públicos a partir de la participación de los actores sociales. En este sentido, la toma de decisiones es producto de la inclusión de todos los actores implicados en asuntos que les concierne (Rosas, 2008). La responsabilidad, la transparencia, la rendición de cuentas y sobre todo la participación y la eficiencia de las acciones públicas son pilares de la capacidad institucional.

Como se observa, el concepto de capacidad institucional ha variado a lo largo del tiempo, no se cuenta con "el concepto" a comprender y mucho menos se expresa en los mismos referentes empíricos. Cada concepto y sus referentes son históricos y dialógicos, ya que se construyen entre diálogos y debates. Por ello comprenderlos implica comprender y entender su génesis y las oposiciones y afinidades que expresan. Es así que las primeras nociones son posturas gerencialistas que se distinguen por abordar la capacidad institucional desde una visión reduccionista, que se limita a la capacidad del aparato administrativo público, sin considerar los atributos o factores del entorno, por lo que el conocimiento y análisis de la capacidad institucional es limitativo. Al pasar de los años y con los cambios históricos aquí planteados, esta perspectiva se superó y se pasó a una concepción donde la capacidad no se restringe al aparato organizacional, a aspectos técnico-administrativos, sino que también 
se refiere a la relación entre el ámbito organizacional y el político, asume una vinculación entre los cuadros técnico-burocráticos, de los grupos de interés, su entorno y sociedad. Sin embargo el asumir concepciones más amplias de la capacidad institucional ha hecho casi imposible diferenciarla de otros conceptos o bien asumirlos como sinónimos, es el caso de "desempeño gubernamental", "desarrollo institucional» o «buen gobierno".

\section{RE-SIGNIFICACIŌN DEL CONCEPTO}

La capacidad institucional como concepto se plantea no sólo con la expresión de un término (palabra) sino conlleva significados declarados por definiciones, los cuales no son únicas sino que se han resignificado. En este momento se cuentan con múltiples definiciones de la capacidad institucional, las cuales, para fines analíticos y expositivos, se clasifican en cuatro grupos: 1) aquellas definiciones que aluden a la capacidad como potencial para cumplir tareas (capacidad indicada), 2) otras que refieren a la capacidad como la actuación del gobierno o su desempeño (capacidad efectiva), 3) unas más que la asumen como las habilidades producidas (capacidad como producto), y por último 4) aquellas que la refieren como los esfuerzos por mejorar la actuación gubernamental o su desempeño (capacidad como proceso). Cada una de ellas, plantean distintos referentes empíricos y formas de pensar el concepto.

Entre las definiciones que hacen alusión a una capacidad indicada, destaca la propuesta de Migdal (1998) que la define como la capacidad que poseen los líderes estatales de utilizar los órganos del Estado con el fin de que se cristalicen sus decisiones en el seno de la sociedad. En esta misma línea está Sikkink y el Centro Latinoamericano de Administración para el Desarrollo (CLAD). Sikkink (1993) la define como la eficacia administrativa del aparato estatal para instrumentar sus objetivos oficiales; y el CLAD como «una condición esencial para que los países latinoamericanos enfrenten en forma más exitosa los problemas de redemocratización, del desarrollo económico y de la distribución de la riqueza" (1997: 9). También se destaca la propuesta de Oszlak y Lattuda y Nogueria. Oszlak (2004) la entiende como la disponibilidad (¿qué hay?, ¿cuánto hay?, ¿de qué calidad?) y aplicación efectiva de los recursos humanos (¿trabajan de acuerdo a lo esperado o no?), materiales y tecnológicos que posee el aparato administrativo y productivo del Estado para gestionar la producción de valor público, sorteando las restricciones, condiciones y amenazas de su contexto. Para Lattuda y Nogueira la asumen como "las 
posibilidades de acción que tiene este complejo institucional [aparato estatal] a través de diferentes políticas públicas, programas, marcos institucionales y demás instrumentos" (2011: 31).

Todos estos planteamientos centran su atención en la habilidad del aparato estatal y en las características técnico-burocráticas del aparato administrativo del Estado y no dan importancia a la relación que se pueda establecer entre el aparato estatal y la sociedad, tampoco a otros atributos o factores del entorno.

Con respecto a las definiciones que se ubican en la capacidad efectiva, sobresale la contribución de Repetto (2004) que la asume como la aptitud de las instancias gubernamentales de plasmar a través de políticas públicas los máximos niveles posibles de valor social. Para este autor es más importante el para qué que el cómo.

En esta misma línea están las propuestas de Fukuda-Parr y colegas (2002) y Acuña y Chudnovsky. Los primeros autores la definen como la habilidad de las instituciones para desempeñar sus funciones, resolver problemas y fijar y lograr objetivos. Acuña y Chudnovsky (2013) definen capacidad institucional como la habilidad que tienen las reglas para incentivar comportamientos y generar horizontes que trasciendan el corto plazo, mediante la resolución de problemas de coordinación y la estructuración, absorción y regulación de conflictos en un ámbito y un momento dados. Estas definiciones se caracterizan por tomar como punto central la efectividad de la actuación gubernamental en la solución de los problemas públicos, dejando de lado criterios igualmente importantes, como la eficiencia, la justicia y la transparencia.

Por otro lado, se encuentran otras definiciones que aluden a una capacidad como producto, es el caso de Hilderbrand y Grindle (1997), Land (2000), Burns (2005) y Ospina (2002) que la definen como la habilidad de desempeñar tareas apropiadas con efectividad, eficiencia y sustentabilidad. Savitch (1998) la entiende como la habilidad de las organizaciones para absorber responsabilidades, operar más eficientemente y fortalecer la rendición de cuentas. Si bien todos estos planteamientos ponen especial énfasis en los resultados de la organización, como en la capacidad efectiva, ahora se pone el acento en la efectividad guiada por los principios y valores de la nueva gestión pública. La eficacia, la eficiencia, la transparencia y la orientación al usuarios, son ejemplo de ello (Aguilar, 2010; Guerrero, 2004; Martínez, 2009 y Cejudo, 2011). No así la justicia o la equidad.

Otras definiciones se refieren a la capacidad como proceso, es decir, como los esfuerzos por mejorar la actuación gubernamental o su desempeño. En esta línea se encuentran Weiss (1998) que hace énfasis en la capacidad 
transformativa del Estado, entendiéndola como la habilidad para adaptarse a los choques y las presiones externas; Chávez y Rayas que la entienden como el "desarrollo de la estructura de toda institución que le permita asumir sus responsabilidades de manera ordenada y coordinada, en el corto, mediano y largo plazo. Es decir, la eficacia y eficiencia de los procesos institucionales, considerando la realización de las tareas y su grado de cumplimiento" (2006: 2). En esta misma línea se encuentra Willems (2003) que la define como la habilidad de las instancias gubernamentales de mejorar las funciones, de resolver problemas y especificar y lograr objetivos. Del mismo modo la asume como la habilidad de movilizar y/o adaptar sus instituciones para dar respuesta a un problema público. Rosas (2008 y 2015a) la asume como la habilidad de las instancias gubernamentales de mejorar el desempeño de las funciones, de resolver problemas y especificar y lograr objetivos; así como de movilizar y/o adaptar sus instituciones para dar respuesta a nuevos problemas públicos.

Bajo esta postura no se puede hablar de una carencia de capacidad institucional. Se parte del principio de que toda organización gubernamental cuenta con una capacidad para la atención de aquellos problemas que son su responsabilidad; pero si se puede presentar un desarrollo de capacidad limitado, lo que repercutirá de manera directa en la eficacia y eficiencia de las políticas públicas que decida llevar adelante. Por ello se busca que las organizaciones gubernamentales se adapten y mejoren sus funciones y así afrontar eficaz y eficientemente aquellos problemas públicos que son su responsabilidad.

Hasta aquí se concluye que el concepto de capacidad institucional cuenta con múltiples significados, ninguna definición aquí planteada es «la definición» a comprender. La manera como se defina determinará sus referentes observables, delimitará qué está incluido en él, y por lo mismo que está excluido, qué características, propiedades o atributos considerar. La definición que se asuma establecerá la observabilidad del concepto en un momento histórico-temporal y en problemas públicos específicos.

\section{CAPACIDAD INSTITUCIONAL ¿PARA QUÉ?}

Más allá de la definición conceptual y de los aspectos que se consideran para su análisis emerge la pregunta ¿capacidad para qué? La respuesta a esta interrogante se puede responder desde diferentes ámbitos y considerando los fines. Partiendo desde lo público y asumiendo los fines que debe perseguir el Estado y sus agencias estatales, se afirma que no se puede desarrollar 
o fortalecer la capacidad institucional en abstracto, o en general, desligada de las responsabilidades que deben ser asumidas y cumplidas. Al respecto Bertranou afirma que "los fines asignados al Estado y sus unidades... no son permanentes, más allá de que puedan entenderse algunas funciones como sustitutivas del Estado como tal. En la medida en que las finalidades son contingentes, las capacidades a desarrollar deben establecerse en función de estas particularidades" (2015: 41).

Actualmente una de las funciones del Estado y sus agencias estatales es orientar sus esfuerzos a la atención eficaz y eficiente de los problemas públicos y lograr atenerlos con políticas exitosas. Sin embargo el éxito o fracaso de toda política pública depende, en gran medida, de las capacidades reales con las que cuentan esas agencias para obtener resultados positivos en relación a sus intervenciones. Depende de las capacidades necesarias o suficientes para atender los problemas públicos.

Capacidades que no son las mismas para todos los problemas, sino que se construyen y fortalecen a partir de las particularidades de cada asunto público, considerando las causas, consecuencias, ventajas e inconvenientes identificadas, la manera como se explican, los efectos buscados y el nivel de entendimiento empírico y conceptual que tenga sobre ellos. Por ejemplo, en el caso del cambio climático si la agencia estatal explica la variación climática a través de los múltiples sistemas ecológicos y humanos y de las variables ambientales, económicas, sociales, políticas, tecnológicas y culturales que se vinculan mutuamente para generar efectos en el clima; entonces el gobierno tendrá que construir o desarrollar una capacidad institucional que le permita trastocar todos estos aspectos. En cambio una definición limitada a un determinado sistema o a ciertas variables disminuye drásticamente las posibilidades de desarrollo de su capacidad y en consecuencia limita la atención del asunto público.

Aunado a lo anterior no será la misma capacidad institucional que se construya si el gobierno reconoce un problema como un asunto más de su acción, como un objeto más sobre el que ha decidido a actuar; que aquella que resulte si lo asume como un problema público y lo coloca entre los asuntos prioritarios y urgentes de su agenda, y por lo tanto pone en marcha toda su maquinaria de información, análisis, concertación, legislación, movilización, recursos económicos y operación para atender, definir y tratar el problema a través de acciones.

Por ello, la capacidad institucional no está dada, ni es homogénea para todos los problemas públicos. Como se expondrá en apartados siguientes, la existencia de las capacidades debe ser comprobada e interpretada en cada 
asunto público, en un tiempo determinado y en un espacio y escala en particular. Son específicas a un contexto histórico-temporal. En este sentido, se cuentan con estudios que explícitamente analizan la capacidad en función de alguna problemática particular, Rosas (2015a) sobre cambio climático, Bertranou (2013) en torno de seguridad vial, Oszlak (2005) sobre regulaciones en telecomunicaciones y problemas agropecuarios, Oszlak, Felder y Forcinito (2000) en torno a regulaciones públicas, Repetto (2003 y 2007) sobre política social, Alonso (2007) en cuestiones de salud, y Depetris (2011) analiza la capacidad para la lucha contra el narcotráfico, entre otros estudios. Cada uno de ellos se caracteriza por proponer ciertos referentes empíricos, llámense componentes, dimensiones, factores o indicadores que permiten evaluar la capacidad institucional construida para cada asunto público.

Pero ¿cómo se ha analizado la capacidad institucional? y ¿qué categorías se han utilizado para que sea fácilmente reconocible? Preguntas que se responden en la siguiente sección.

\section{EJES DE ANÁLISIS}

Hasta aquí se ha expuesto que la capacidad institucional como concepto

se ha planteado con la expresión de más de un término y su correspondiente significado (definición). Ahora se sostiene que como concepto también es fundamental expresar sus referentes empíricos que permitan analizarlo mediante la observación en cada asunto público, en un tiempo determinado y en un espacio y escala particular. Es así que actualmente se cuenta con una diversidad de propuestas que proponen aspectos observables, llámense componentes/ dimensiones, factores o indicadores, que permiten demostrar la presencia de capacidad institucional o bien analizar su desarrollo o fortalecimiento. Algunas de ellas no alcanzan un nivel operacional, es decir, "no logran plantear un concepto transferido y reducido a sus propiedades observables y definido por las operaciones que lo verifican" (Sartori, 2015: 66); pero si representan una guía orientadora para valorarla. Otras propuestas plantean analizar la capacidad institucional a partir de ciertos referentes empíricos pero sin validar su elección. Es así que tenemos propuestas que por sus características no son integrales y carecen de una estrategia que les permita validar la selección de sus referentes para evaluar la capacidad institucional.

Propuestas que se pueden clasificar en minimalistas y maximalistas (Munck y Verkuilen, 2002). Las primeras restringen el análisis a unas pocas dimensio- 
nes/componentes y atributos corriendo el riesgo de no considerar aquellas que pueden ser importantes; y las segundas tienden a incluir numerosos atributos y miradas multidimensionales que en ocasiones hacen difícil el estudio. Dentro del primer grupo se destaca contribuciones que se limitan al aparato organizacional, a aspectos técnico-administrativos. Es el caso de Castro que identifica tres componentes: a) la capacidad de planificación como la habilidad de definir sus objetivos y metas (planificación estratégica), así como del desarrollo e implementación de acciones específicas para alcanzarlos considerando la presupuestación; b) capacidad de ejecución como la habilidad de las entidades para llevar acabo los gastos e inversiones previstas de acuerdo al presupuesto aprobado; así como la habilidad para que las entidades entreguen los bienes y servicios a través de los cuales espera lograr los resultados previstos con la implementación de sus políticas y programas, y c) eficiencia operacional como la habilidad para hacer un uso adecuado de insumos para la entrega de bienes y senvicios por parte de las entidades, principalmente se toma en cuenta el recurso humano y los recursos financieros (Castro, 2011).

De igual forma se encuentra la contribución de Charles Tilly (1992), que identifica dos dimensiones centrales para la comprensión de la capacidad estatal, la fiscal y la coercitiva, argumentando que las mismas surgen como un subproducto de los esfuerzos que realizan los gobiernos nacionales para acceder a los medios necesarios para hacer la guerra y, a través de ella, para expandir a los estados-nación.

En este mismo grupo se identifican propuestas que abren su análisis a la relación que se establece entre el Estado y la Sociedad. Aquí destaca Evans (1996) que con su idea de autonomía arraigada (embedded autonomy), identifica la coherencia interna y conectividad externa. Respecto a la "coherencia interna", Evans, jerarquiza la importancia que tiene para el funcionamiento estatal la existencia de cuadros burocráticos que se caractericen por ser expertos, que se recluten a través del mérito y que existan oportunidades para obtener promociones y ascensos de una carrera profesional de largo plazo. De igual forma, sostiene que la capacidad del Estado dependía de que la burocracia fuese una entidad corporativamente coherente, y de que los individuos viesen en la consecución de las metas corporativas la mejor manera de promover su interés personal.

En relación a la "conectividad externa", Evans jerarquiza el papel que para el efectivo funcionamiento del Estado tiene el vínculo entre los funcionarios públicos y los grupos sociales. De ahí que se demanda un Estado más bien "enraizado (o encastrado) en la sociedad que aislado" (Evans, 1996: 536). Cuando los lazos entre "el adentro" y "el afuera" dan lugar a interacciones 
virtuosas entre los actores involucrados el aparato estatal aumentará su capacidad para instrumentar las políticas públicas.

También destaca la contribución de Grindle, Palermo, Evans, Alonso, Tobelem, Oszlak y Orellana y Repetto. Con respecto a Grindle (1997) su propuesta delimita el análisis de la capacidad estatal a cuatro componentes: a) la capacidad administrativa como la habilidad de los estados de llevar adelante la distribución de bienes y servicios; b) la capacidad técnica como la habilidad de manejar las políticas macro económicas y determinar opciones de dichas políticas; c) la capacidad política, para responder a demandas sociales y resolver conflictos; y d) la capacidad institucional para fortalecer las reglas de juego.

Palermo (1998) reduce el análisis de capacidad institucional a dos dimensiones: a) institucionales: que corresponde a las reglas de juego formales e informales socialmente compartidas que moldean las interacciones de los hombres en determinado contexto, estas reglas establecen la forma en que se dan los incentivos, generando "expectativas acerca de los derechos y obligaciones que los jugadores pueden presumir en una determinada transacción social y dando la posibilidad de construir confianza en la medida en que cada uno se comporta de acuerdo a las expectativas; y b) organizacional: corresponde a los jugadores —agentes públicos - y la manera en que están organizados para cumplir objetivos. Cuando los jugadores se benefician de las reglas de juego imperantes, procuran conservarlas y estabilizarlas lo más posible. Por el contrario, cuando el beneficio que se obtiene de ellas es nulo o no está de acuerdo a las expectativas, tratarán de cambiarlas.

Evans (1996), Alonso (2001), Tobelem (1992), Oszlak y Orellana (2001), Repetto (2001) y Rosas (2015a) reducen su análisis a dos componentes: a) capacidad administrativa y b) capacidad política, y son con los que en mayor medida existe un consenso. Los atributos asociados a estos componentes son variados, por ejemplo para Repetto son: «coordinación, flexibilidad, innovación, calidad, sostenibilidad, evaluabilidad, eficiencia, eficacia, accountability, legitimidad, equidad pertinente" (2004: 20-21).

Las propuestas de estos autores coinciden en que las capacidades institucionales no se limitan única y exclusivamente a las características y actuación del aparato burocrático, sino también a las arenas políticas que se configuran y en las que se procesan intereses e ideologías tanto internas como externas al entramado organizacional estatal. Es por ello que el Estado y sus dependencias deben ser analizados en su doble carácter de aparato institucional y de relación social. No es suficiente considerar las habilidades técnico-burocráticas y la actuación de organizaciones administrativas del aparato estatal requeridas 
para instrumentar los objetivos oficiales, sino también es necesario asumir la importancia que tiene la constitución y acción de los actores sociopolíticos involucrados en un determinado campo de acción pública. Se debe reconocer que las estructuras institucionales no son neutrales con respecto a la constitución de los intereses y preferencias de los actores no gubernamentales.

En el segundo grupo destacan los aportes de Tobelem (1992), que sugiere seis dimensiones para el análisis de la capacidad institucional: los individuos participantes en las agencias involucradas; las políticas de personal y sistemas de recompensas, la capacidad financiera y física de las agencias; la estructura organizacional interna y la distribución de funciones; las relaciones interorganizacional y el sistema de leyes, reglas, normas y «reglas de juego".

Hilderbrand y Grindle (1997) proponen cinco aspectos a considerar para el análisis de la capacidad: los recursos humanos dentro de cada organización, la organización misma, el conjunto de organizaciones con las cuales ella se relaciona para funcionar efectivamente, el contexto institucional del sector público, que incluye las reglas que enmarcan el accionar gubernamental: recursos, atribuciones, competencias, etc., y finalmente el entorno económico, político y social dentro del cual se enmarca el sector público.

De igual forma Forss y Venson (2003), recuperando la propuesta de Grindle propone cinco aspectos a considerar: 1) el individuo; 2) la organización; 3) la red de organizaciones; 4) la gobernación pública; y 5) las normas, valores y prácticas sociales.

Oszlak y Orellana (1993) identifican seis déficit de la capacidad institucional, las cuales pueden representar aspectos a tomar en su análisis, a saber: 1) Déficits relacionados con la existencia de normas, leyes y "reglas de juego» que condicionan o impiden la ejecución de tareas; 2) Déficits relacionados con la capacidad financiera y física de las agencias ejecutoras, 3) Déficits relacionados con las relaciones interinstitucionales; 4) Déficits relacionados con la estructura organizacional interna y distribución de funciones; 5) Déficits relacionados con las políticas de personal y sistemas de recompensas y; 6) Déficits relacionados con la capacidad individual de los participantes en las agencias involucradas en el proyecto.

Por su parte el PNUD (2010) identifica cuatro "pilares clave» del desarrollo de capacidades, a partir de los cuales ensaya una suerte de enumeración de indicadores que estarían dando cuenta de su presencia: a) arreglos institucionales: procesos racionalizados, clara definición de funciones y responsabilidades, mecanismo de valoración de méritos, mecanismo de coordinación; b) liderazgo: formulación clara de la visión, estándares de comunicación, herramientas de 
gestión, entre otros; c) conocimiento: mecanismos para vincular investigación, demanda y suministro; estrategias para captar y retener cerebros, mecanismos y herramientas para la gestión del conocimiento; d) rendición de cuentas: sistemas de auditoría y normativas de prácticas, mecanismo de planificación participativa, mecanismo de retroalimentación de las partes, entre otros.

Chudnovsky (2014) identifica cinco dimensiones: 1) capacidad organizacional que se refiere a la concordancia entre las metas de la organización y los recursos, procesos, infraestructura con la que cuenta; 2) capacidad presupuestaria que alude tanto al monto como a la organización del presupuesto; 3) capacidad del servicio civil que se enfoca a las características técnicas del servicio civil y formación e idoneidad de los recursos humanos; 4) capacidad de alcance que alude a la capacidad de llegar al público objetivo en el territorio $y$ al bloqueo de resistencias de grupos organizados, tanto sociales como económicos; y 5) capacidad política: que se refiere a la construcción de poder y reconocimiento de otras agencias de igual jerarquía dentro de la administración pública como de autoridades políticas superiores a las de la agencia.

La mayoría de las contribuciones se caracterizan por analizar la capacidad institucional a través de indicadores, organizados en componentes, que valoran tanto las habilidades y competencias de los recursos humanos y organizacionales, como la relación que se establece entre Estado y sociedad para la atención de los problemas públicos. Las propuestas analíticas no establecen una organización jerárquica que permita observar diferenciadamente los recursos humanos, la organización gubernamental y la relación Estado-sociedad, principalmente aquellas referidas al ejercicio al ejercicio real del poder.

Por ello, y con base a las propuestas de Evans (1996), Grindle (1997a), Forss y Venson (2003), Alonso (2001), Tobelem (1992), Oszlak y Orellana (2001) y Repetto (2004), y asumiendo a la capacidad institucional como un proceso que no se limita al aparato organizacional sino que también a la relación entre el ámbito el ámbito organizacional y todos aquellos actores que de manera directa o indirecta están vinculados con los problemas públicos, se propone una metodología que se construye a partir de componentes, niveles e indicadores. Un método más acotado empíricamente y que permite centrar el análisis en la relación que existe entre los cuadros técnico-burocráticos, las organizaciones estatales, los grupos de interés, su entorno y sociedad, ${ }^{2}$ a través de dos componentes: capacidad administrativa y capacidad política; tres niveles: el micro (el individuo), el meso (la organización) y el macro (el contexto institucional), que hacen parte de los componentes y un conjunto de factores institucionales que son caracterizados por atributos observables empíricamente y que son agrupados en los niveles (ver tabla 1). ${ }^{3}$ 


\section{Tabla 1.}

Propuesta metodológica para evaluar la capacidad institucional

\begin{tabular}{lll}
\hline Componente & Nivel & Factor \\
\hline $\begin{array}{l}\text { Capacidad } \\
\text { administrativa }\end{array}$ & $\begin{array}{l}\text { Micro } \\
\text { (recursos humanos) }\end{array}$ & Recursos humanos \\
\cline { 2 - 3 } & Meso & \\
& (organización) & $\begin{array}{l}\text { Reconocimiento público y prioritario } \\
\text { Autoridad } \\
\text { Cooperación } \\
\text { Coordinación }\end{array}$ \\
\hline Capacidad & Macro & $\begin{array}{l}\text { Recursos económicos } \\
\text { política }\end{array}$ \\
\hline
\end{tabular}

La capacidad administrativa está referida a "las habilidades técnico burocrático del aparato estatal para instrumentar sus objetivos oficiales" (Rosas, 2015a: 53) e incluye al nivel micro (recursos humanos) y al nivel meso (la organización). El nivel micro, tiene como objeto de evaluación la unidad más pequeña, esto es el factor de los recursos humanos o bien los individuos con los que cuenta la organización gubernamental para cumplir con un fin específico. Este nivel asume que las habilidades y aptitudes de los individuos dentro de cada organización determinan la actuación de éstos en sus funciones; por ello los individuos son la base para el éxito de cualquier acción o política. En este sentido es que considera que los atributos propios del factor recursos humanos son: número de personas dedicadas a la gestión y coordinación de las acciones orientadas a atender el problema, tipo de personal contratado (directivo u operativo), tiempo de dedicación, tipo de contratación e idoneidad del perfil (formación vs actividades laborales desempeñadas).

Por su parte, el nivel meso alude a la organización, aquí se analiza el funcionamiento general de las entidades ejecutoras y se evalúa la eficacia y eficiencia de las mismas. Los factores que se proponen para analizar este nivel son: el reconocimiento público, la autoridad, la cooperación y la coordinación y los recursos económicos con los que cuenta. Los atributos de estos se muestran en la tabla 2. 
Tabla 2.

Factores y atributos del nivel meso

\begin{tabular}{|c|c|}
\hline Factor Institucional & Atributos \\
\hline $\begin{array}{l}\text { Reconocimiento } \\
\text { público y prioritario } \\
\text { del problema o } \\
\text { cuestión pública }\end{array}$ & $\begin{array}{l}\text { Marco legal específico que establezca responsabilidades, niveles de } \\
\text { coordinación, cooperación y autoridad en determinada cuestión. } \\
\text { Existencia de organizaciones gubernamentales encargadas del problema, } \\
\text { con la autoridad para tomar decisiones. } \\
\text { Asignación suficiente de recursos económicos para el cumplimiento de } \\
\text { las decisiones (por ejemplo: el diseño y la implementación de acciones, } \\
\text { la capacitación del personal). } \\
\text { Asignación suficiente de recursos humanos para el cumplimiento de las } \\
\text { decisiones (capacitados). } \\
\text { Existencia de programas gubernamentales enfocados específicamente } \\
\text { a determinada cuestión. }\end{array}$ \\
\hline Recursos económicos & $\begin{array}{l}\text { Presupuesto público destinado para sueldos y salarios. } \\
\text { Presupuesto público para la coordinación de acciones enfocadas a } \\
\text { determinado asunto. } \\
\text { Existencia de un programa operativo para implementar las decisiones. }\end{array}$ \\
\hline $\begin{array}{l}\text { Cooperación y } \\
\text { coordinación }\end{array}$ & $\begin{array}{l}\text { Cooperación y coordinación intergubernamental. } \\
\text { Cooperación y coordinación intersectorial. } \\
\text { Cooperación y coordinación sustentada en objetivos comunes. } \\
\text { Cooperación y coordinación sustentada en una visión común e integral. }\end{array}$ \\
\hline Autoridad pública & $\begin{array}{l}\text { Existencia de marco legal que establezca responsabilidades. } \\
\text { Existencia de actores gubernamentales responsables. } \\
\text { Otorgamiento de poder a los responsables. } \\
\text { Capacidad de Influir en otras organizaciones. }\end{array}$ \\
\hline
\end{tabular}

Por otro lado, la capacidad política hace referencia a la interacción política que, enmarcada en ciertas reglas, normas y costumbres, establezcan los actores del Estado y el régimen político con los sectores socioeconómicos y con aquellos que operan en el contexto internacional. El análisis se centra en dos planos, el primero se sitúa en la interacción que establecen los propios actores del Estado ubicados en los poderes de gobierno (ejecutivo, legislativo y judicial) y niveles de gobierno, por ejemplo para el caso de México se enfocaría al nivel federal, estatal y municipal y en Argentina a las provincias, municipios y comunas. El segundo nivel de análisis se refiere a las relaciones del Estado con diversos individuos y grupos de la sociedad civil que luchan 
por involucrarse activamente en los procesos de políticas públicas. "Al forjar la política, los actores sociales y el Estado interactúan; los unos requieren del otro. La interacción de los actores sociales es afectada por las reglas y las instituciones por las cuales actúan. Pero el efecto de las reglas y de las instituciones depende de quién trate de usarlas y con qué propósiton (Gourevitch, 1993: 268). Es así que en esta dimensión está presente el nivel macro de la capacidad institucional, aquí se involucra a las acciones del Estado, los partidos políticos, los grupos de interés y las organizaciones no gubernamentales involucradas en el proceso de las políticas públicas. Este nivel se caracteriza por reconocer que los individuos y las organizaciones están inmersas en redes de organizaciones y a su vez éstas se encuentran vinculadas en un contexto institucional más amplio, es decir, en lo que se llama "gobernación pública" (Forss y Venson, 2003). Ésta hace alusión a cómo se gobierna; por ejemplo el proceso de toma de decisiones en los problemas de la política; la habilidad de los ciudadanos, grupos y asociaciones en participar; la transparencia en la información, cómo y para que participan los ciudadanos, grupos y asociaciones. Aquí se problematiza, por ejemplo, en torno a ¿quiénes participan? ¿Cómo participan? ¿Existe una voluntad política entre los actores para participar? y ¿existen negociaciones entre actores?

Cada factor institucional posee por sí mismo un grado específico de injerencia en la capacidad institucional, pero que ubicados en la actividad gubernamental se relacionan y se determinan mutuamente, dando origen a cierta capacidad institucional. Las relaciones que se establecen entre los factores institucionales ubicados en "el adentro" (nivel micro y meso) y "el afuera" (nivel macro) pueden dan lugar a interacciones virtuosas que se reflejan en la presencia misma de la capacidad institucional, así como en su propio desarrollo o fortalecimiento, pero también pueden generar déficit de las capacidades institucionales.

Como se puede observar este proceder metodológico se distingue por plantear una disección analítica sustentada en una organización jerárquica que permite ordenar los referentes observables en aquellas dimensiones constitutivas que describen y explican la capacidad institucional y asumir que todos los referentes planteados son incluidos en los elementos que están por encima de ellos.

Considerar estos componentes y niveles permite pasar de una concepción estática y cerrada a una dinámica en donde el desarrollo y/o fortalecimiento de la capacidad institucional se da en relación con otros, en este caso con el Estado, la sociedad, el sistema político y en general con todos los actores que de manera directa o indirecta están vinculados con los problemas públicos. 
También permiten reconocer que los componentes, los niveles y los factores institucionales se determinan mutuamente, es decir, lo que sucede en uno es claramente influido por los otros y que la capacidad institucional no se construye o fortalece única y exclusivamente con un solo factor, sino por múltiples factores. Los aquí propuestos fueron considerados como los que ejercen mayor influencia en la capacidad institucional; sin embargo se reconocen que se dejaron de lado otros que podrían ser igualmente importante para ciertos asuntos públicos y que pueden escapar del control del Estado, por ejemplo para el caso del cambio climático es importante considerar la existencia de políticas coherentes e integrales de largo plazo donde estén presentes todos los sectores involucrados en el problema, el flujo adecuado de la información requerida para atender el problema (información completa, oportuna y confiable), el entorno económico, político y social y la participación del gobierno en las negociaciones internacionales.

Por ello cualquier estudio sobre la capacidad institucional debe asumir, por una parte, que existe una diversidad de factores que la determinan y que se ubican en sus diferentes niveles y componentes, que existen relaciones entre ellos y que "no se crean de la noche a la mañana ni por decisiones ad hoc; tampoco son el producto de una mera reorganización de las estructuras, sino el fruto de un largo proceso histórico de construcción institucional» (Sikkink, 1993: 2). Por otra, que el estudio de algunos factores puede ser más complejo que otros y cada uno requiere de un análisis exhaustivo. Así queda reflejado en la diversidad de estudios que analizan la capacidad institucional para enfrentar diversos problemas públicos (Grindle, 1997a; Sikkink, 1993; Evans, 1996; Oszlak, 2004; UNICEF, 1999; Tobelem, 1992; Oszlak y Orellana, 2001; Palermo, 1998; Alonso, 2007; Camou, 2001; Repetto, 2003 y 2004; y Nelissen, 2002).

Cabe mencionar que es una propuesta que ha sido utilizada, con algunas variantes, para evaluar la capacidad institucional que los gobiernos desarroIlan para atender problemas sociales y ambientales (Celestino, 2014, Rosas, 2015a y 2015; Díaz, 2017; Moreno, 2018). Esto debido a que algunos factores considerados son catalogados como tradicionales, ya que comúnmente influyen en la capacidad que tiene las instancias gubernamentales para resolver problemas públicos. Son aspectos que toda dependencia estatal necesita para atender cualquier asunto público (e. g. recursos humanos, recursos económicos, cooperación). Existen otros que son considerados como nuevos (Willems, 2003; y Willems y Baumert, 2003), esto depende del tipo de problema público que se busca atender; por ejemplo en la política climática se consideran la cooperación y coordinación entre niveles de gobierno, sectores 
y actores públicos y privados, el conocimiento e información para la toma de decisiones, la participación de actores sociales y privados en las decisiones sobre el tratamiento de los temas de vulnerabilidad, adaptación y mitigación como factores nuevos e importantes para el fortalecimiento de la capacidad institucional que se construye para atender el problema de cambio climático (Rosas, 2015a y 2015b). En cambio, los factores institucionales específicos que requieren desarrollar las dependencias estatales para que cuenten con las capacidades necesarias para fomentar el deporte social son: reconocimiento público y prioritario que se le asigna al deporte como un derecho y como medio para alcanzar objetivos del desarrollo social por parte de la autoridad gubernamental, intervenciones públicas, con un adecuado entendimiento del fomento del deporte social y con enfoque de acciones afirmativas dirigidas a generar el mayor valor social posible; y cooperación y coordinación que tiene la organización gubernamental encargada de la política deportiva con diversos actores interesados en el asunto, poseedores de diversas perspectivas y recursos, para garantizar que un mayor número de personas tengan acceso a la práctica del deporte social (Moreno, 2018).

Aunado a los componentes y niveles, también se debe considerar para su análisis una perspectiva espacial, asumiendo que el desarrollo o fortalecimiento de la capacidad institucional se da en un territorio específico, en un espacio particular, el cual «no está constituido por una colección de cosas ni por un agregado de información (sensorial) ni por un paquete vacío parcela de varios contenidos, que es irreductible a una forma impuesta, a un fenómeno, a las cosas o a una materialidad física" (Lefebvre, 1991: 27), sino es una construcción social. Es un espacio producido por las relaciones de los actores ubicados no sólo en la esfera gubernamental sino también en la sociedad. Actores que no son homogéneos, sino diferentes por el tipo de actividad que realizan y por las redes de actividades que generan al interior de los espacios y por las relaciones sociales que construyen (Massey, 1991: 30).

Por lo que no existe un espacio unívoco, ni mucho menos fijo e inmóvil donde se pueda analizar la capacidad institucional, el análisis se puede centrar en múltiples espacios, se puede tomar a un país, a un estado, a un municipio, a una ciudad, a una zona metropolitana, a una megalópolis, entre otros. Cada uno de estos espacios presentan problemas específicos, contextos diferentes, diversidad de actores y actividades y multiplicidad de relaciones sociales.

Además del espacio, hay que considerar, como bien lo señala Bertranou, las funciones que un organismo debe cumplir o los objetivos que el mismo debe alcanzar. «No puede analizarse la capacidad estatal en abstracto, sino en 
relación con los fines e incluso metas que el organismo analizado debe cumplir" (2011: 32). En este sentido la capacidad institucional se pone en juego en varios ámbitos y niveles de intervención estatal, con sus correspondientes desagregaciones en los niveles de áreas, políticas, programas y proyectos.

Cada política pública se desarrolla en el marco de cierta capacidad institucional. Algunas políticas tienen un carácter repetitivo y permanente en el largo plazo que permiten recrear de forma permanente la intervención pública, y en por ende una mejora continua de la intervención pública y de su capacidad institucional. Otras donde el avance en los efectos esperados están en función del desarrollo mismo de los proyectos que las integran y no se cuenta con la experiencia y la oportunidad de la experimentación; por lo que el conocimiento sobre con qué cuentan las organizaciones gubernamentales y qué características tienen resulta fundamental.

\section{REFLEXIONES FinALES}

Resulta evidente que los debates acerca de la capacidad institucional no están agotados. La revisión que se hace de la bibliografía sobre este concepto sugiere que éste ha sido utilizado de manera bastante arbitraria por diferentes autores que resulta casi imposible diferenciarlo de otros conceptos. De igual forma se mostró que existen múltiples propuestas para analizarlo, propuestas que se caracterizan por proponer aspectos observables de la capacidad institucional, llámense componentes, dimensiones, factores o indicadores. Son contribuciones que permiten realizar un análisis empírico desde una visión reduccionista (minimalista) o bien asumiendo una concepción dinámica en donde el desarrollo y/o fortalecimiento de la capacidad institucional se da a partir de una relación entre el Estado con la sociedad, el sistema político y los actores que están vinculados con los asuntos públicos (maximalista). Otra limitante observada en las contribuciones es que algunas no asumen, clarifican o validan su método.

Si bien la selección del método para el análisis de la capacidad institucional depende del investigador, también está en función de lo que se quiera identificar en un tiempo determinado y en un espacio y escala en particular; así como de los asuntos públicos que deben ser atendidos por el Estados y sus agencias estatales. No es la misma capacidad institucional para todos los problemas públicos, dicha capacidad no está dada ni es homogénea para todos los problemas, es así que dicha capacidad es específicas a un 
contexto histórico-temporal y a cada problema público. Por lo que no existe un método univoco y valido, pueden existir varios, aquí se propone uno que se distingue por plantear una organización jerárquica que ordena y relaciona los referentes observables que describen y explican la capacidad institucional. Es un método que asume que dicha capacidad está en función tanto de los aspectos internos de la organización estatal, como también de aquellos que están fuera de ella, algunos de carácter institucional, otros de índole política, sociocultural, económica y/o asociado a la red de organizaciones que con su accionar afectan directa o indirectamente los resultados previstos en relación a sus intervenciones de las dependencias estatales.

Es un método que asume que el problema inicial es identificar la capacidad institucional en un asunto público, por lo que para identificarlo primero se aísla y se distingue de otros asumiendo un espacio y escala en particular y se relaciona con los referentes observables y con lo que le antecede y sucede, con lo que fue y será. Sin embargo se dejaron de lado otros que podrían ser igualmente importantes para ciertos asuntos públicos y que pueden escapar del control del Estado.

\section{NOTAS}

${ }^{1}$ Ejemplo de estas definiciones son presentadas en el estudio que hace la UNICEF (1999).

2 Los componentes y niveles son entendidos como las dimensiones constitutivas de la capacidad que pueden ser observables en una realidad concreta, son los que describen y explican la capacidad institucional.

${ }^{3}$ El método se construyó en dos momentos. En el primero, se realizó una revisión de estudios que han analizado la capacidad institucional de aquellos gobiernos nacionales interesados en atender el cambio climático, así como de los decretos, planes y programas de relacionados a esate tema de algunos gobiernos locales. Esta revisión permitió identificar la recurrencia con que los factores institucionales se presentan en dichos estudios y seleccionar aquellos que estuvieran presentes en más de la mitad de ellos, así como sus atributos y relaciones. De igual forma, dicha selección se realizó tomando en cuenta la opinión de informantes clave, la cual fue recuperada a través de una entrevista semiestructurada. En un segundo momento se validaron estos factores institucionales, sus atributos y relaciones, así como se incorporaron otros que no habían sido tomados en cuenta. La validación e incorporación de factores y atributos se realizó tomando en cuenta la opinión de 30 expertos en el tema. La recuperación de dicha opinión se dio a partir de la aplicación de un cuestionario a través de un ejercicio Delphi. Para una descripción más detallada, ver Rosas y Gil, 2013; y Rosas, 2015. 


\section{BIBLIOGRAFÍA}

Acuña, Carlos y M. Chudnovsky (2013). "Cómo entender las instituciones y su relación con la política" en Acuña, Carlos (comp.) ¿Cuánto importan las instituciones? Gobierno, Estado y Actores en la Política Argentina. Argentina, Siglo XXI-Fundación OSDE.

Aguilar, L. (2010). "El futuro de la gestión pública y la gobernanza después de la crisis». En Frontera Norte, vol. 22, núm. 43, enero-junio, México, El Colegio de la Frontera Norte, A.C.

Alonso, G. (2007). "La reforma del seguro de salud. Política, instituciones y capacidades regulatorias", en Alonso, G. (Ed.), Capacidades estatales, instituciones y política social, Argentina, Prometeo.

Alonso, G.V., O'Donell, G., M. Cárdenas, M.M. Daniel, O. Oszlak, Barbara Geddes, et al. (2015). Capacidades estatales. Diez textos fundamentales, Argentina, Banco de Desarrollo de América Latina.

Alonso, G. (2001). Notas para el análisis de capacidades institucionales en el sector público, Documentos de Trabajo, Buenos Aires, UNSAM.

Banco Mundial (1997). Colombia, estudio sobre la capacidad de los gobiernos locales: más allá de la asistencia técnica en la descentralización y el desarrollo institucional en Colombia, hoy: un análisis a la descentralización. Bogotá Colombia, Escuela Superior de Administración Pública (ESAP).

Bertranou, J. (2011). Análisis preliminar del desarrollo de capacidades estatales en el nivel nacional para la promoción y fiscalización de la seguridad vial, Buenos Aires, Argentina, Ministerio del Interior de la Nación.

(2013). "Creación de agencias especializadas, capacidad estatal y coordinación interinstitucional. El caso de la Agencia Nacional de Seguridad Vial en Argentina", en Revista Perspectivas de Políticas Públicas, № 4, enero-junio, Argentina, UNLa, Lanús.

(2015). "Capacidad estatal: Revisión del concepto y algunos ejes de análisis y debate" en Revista Estado y Políticas Públicas № 4.

Burns, J.R. (2005). Government capacity and the Hong Kong civil service, E. U., Oxford University Press,

Camou, A. (2001). "Estudio preliminar" en Camou, Antonio (comp.) Los desafíos de la gobernabilidad, México, FLACSO México/UNAM/Plaza y Valdés.

Castro, F. (2011). Diseño de un índice de capacidad institucional para la efectividad del gasto público. Informe final, FEDESARROLLO-USAID 
Celestino, E. (2014). "Evaluación sobre capacidad institucional del gobierno mexicano en atención a la vulnerabilidad social con jefatura femenina", Tesis de licenciatura, Universidad Autónoma Metropolitana.

Centro Latinoamericano de Administración para el Desarrollo, CLAD (1997). Una nueva gestión pública para América Latina, Documento del CLAD, http://unpan1.un.org/intradoc/ groups/public/documents/CLAD/UNPAN000161.pdf.

Cejudo, G. (Coord.) (2011). «La nueva gestión pública. Una introducción al concepto y a la práctica». En: Cejudo, G. Nueva Gestión Pública, México, BBAPdf-Siglo XXI.

Chávez, O. e I. Rayas. (2006). "Fortalecer la capacidad institucional. La plataforma para un gobierno exitoso" en Portal de desarrollo, 26 de junio, consultado en Internet: http://www. portaldeldesarrollo.org/gobierno/ recursos. php?idseccion $=272 \&$ idcontenido $=292$.

Chudnovsky, M. (2014). «Régimen político, Estado y Políticas Públicas: el Rol de los Gabinetes en el Desarrollo de las Capacidades Estatales", Tesis doctoral, Universidad Torcuato Di Tella.

Completa, R. (2016). "Capacidad estatal, brechas de capacidad y fortalecimiento institucional", en Análisis Político, nº 29.

Depetris, J. (2011). Deportes Extremos: Construyendo capacidades estatales en la lucha contra el narcotráfico. El caso de la Aduana Argentina (2005-2010). Cuadernos del Instituto AFIP. No 21.

Díaz, D. (2017). "La capacidad institucional del Estado de México para atender el cambio climático", Tesis de licenciatura, Universidad Autónoma Metropolitana.

Evans, M. (1996). "El Estado como problema y como solución" en Desarrollo Económico. Revista de Ciencias Sociales, Enero-Marzo de 1996, vol. 35, n 140, México, Instituto de Desarrollo Económico y Social.

Forss y Venson en Willems, S. y K. Baumert (2003). Institutional capacity and climate actions, France, OECD Environmental Directorate International Energy Agency.

Fukuda-Parr, S., Lopes, C. \& Malik K. (2002). "Overview: Institutional Innovations for Capacity Development", in Capacity for Development, New Solutions to Old Problems, UNDP-Earthscan.

Gourevitch, P. (1993). Políticas estratégicas en tiempos difíciles, México, Fondo de Cultura Económica.

Grindle, M. (1997a). "The good government imperative. Human Resources, organizations and institutions" en Grindle, Merilee S., Getting good government. Capacity building in the public sectors of developing countries, Boston, Harvard University Press. 
(1997b). Getting good government: capacity building in the public sector of developing countries, Harvard Institute for International Development, Boston.

Guerrero, 0. (2004). La Nueva Gerencia Pública. Neoliberaismo en Administración Pública, México, Editorial Fontanera.

Hall, J.S. (2002). "Reconsidering the connection between capacity and governance» en Public Organization Review: A Global Journal 2, Kluwer Academic Publishers.

Hilderbrand, M. y M. Grindle (1997). "Building Sustainable Capacity in the Public Sector: What Can Be Done?" en Grindle, M. Getting Good Government. Capacity Building in the Public Sector of Developing Countries, Boston, Harvard University Press.

Land, A. (2000). Implementing Institutional and Capacity Development: Conceptual and Operational Issues, ECDPM Discussion Paper 14, Maastricht, ECDPM.

Lattuada, M. y Nogueira, M.E. (2011). "Capacidades estatales y políticas públicas. Una propuesta para el abordaje de las políticas agropecuarias en la Argentina contemporánea (1991-2011)" en Estudios Rurales Vol. 1, № 1. Universidad Nacional de Quilmes.

Lefebvre, H. (1991). The production of space, Oxford, Blackwell.

Martínez, J. (2009). "La nueva gerencia pública en México. Una mediación de su intensidad e impactos en las entidades del país". En: Convergencia, vol.16, n 49, enero/abril, Toluca, México, Universidad Autónoma del Estado de México.

Massey, D. (1991). "Las regiones y la geografía" en Ramírez, B., Nuevas tendencias en el análisis regional, México, UAM-Xochimilco.

Migdal, J. (1988). Strong Societies and Weak States, Princeton, Princeton University Press.

Mizrahi, Y. (2003). Capacity Enhacement Indicators: REview of the Literature, Washington, D.C., World Bank Institute.

Moreno, R. (2019). "Alcances y desafíos de la capacidad para el fomento del deporte social. Un análisis del Instituto del Deporte de la Ciudad de México (2012-2018)", Tesis de maestría, Universidad Autonónoma Metropolitana.

Munck, G. y J. Verkuilen (2002). "Conceptualizing and Measuring Democracy: Evaluating Alternative Indices", en Comparative Political Studies, nº 35 (1).

Nelissen, N. (2002). "The Administrative Capacity of New Types of Governance», en Public Organization Review: A Global Journal 2, Kluwer Academic Publishers.

Ospina, S. (2002). "Construyendo capacidad institucional en América Latina: el papel de la evaluación como herramienta modernizadora». Presentado en VII Congreso Internacional del 
CLAD sobre la reforma del Estado y de la Administración Pública, Lisboa, Portugal. 8 al 11 de octubre, Centro Latinoamericano de Administración para el Desarrollo.

Oszlak, 0. (2004). Transformación estatal y gobernabilidad en el contexto de la globalización: un análisis comparativo de Argentina, Brasil, Chile y Uruguay: el Caso Argentino, Propuesta de investigación presentada por equipo de consultores del Centro de Desarrollo y Asistencia Técnica en Tecnología para la Organización Pública.

- (2005). «Políticas sectoriales, transfor-mación estatal y gobernabilidad en la Argentina: de Menem a Kirchner», Ponencia presentada al X Congreso del CLAD, Santiago de Chile.

(2006). "Burocracia estatal: Política y Políticas Públicas», en POSTData Revista de Reflexión y Análisis Político, Vol. XI, Buenos Aires.

Oszlak, O. y E. Orellana (1993). «El análisis de la capacidad institucional: aplicación de la metodología SADCl», versión digital en www.top.org.ar.

- (2001). El análisis de la capacidad institucional: aplicación de la metodología SADCI, Mimeo, Buenos Aires.

Oszlak, O., Felder, R. y Forcinito, K. (2000). La capacidad regulatoria del Estado en Argentina, Buenos Aires, INAP.

Palermo, V. (2008). "Capacidades estatales, instituciones y política social», en Alonso Guillermo V. (editor). Capacidades estatales, instituciones y política social, Buenos Aires, Prometeo Libros.

(1998). Estudio sobre el estado del Estado en Argentina, Documento de Trabajo, Buenos Aires, Fundación Carlos Auyero.

Programa de las Naciones Unidas para el Desarrollo (2010). «Medición de la capacidad», junio de 2010.

Repetto, F. (2003). Los actores de la política social, mimeo, Washington DC.

(2003). "Capacidad estatal: requisito necesario para una mejor política social en América Latina». Ponencia presentada al VIII Congreso del CLAD, Panamá.

(2004). Capacidad Estatal: requisito para el mejoramiento de la Política Social en América Latina. INDES - Banco Interamericano de Desarrollo, Serie de Documentos de Trabajo I-52, julio de 2004.

_- (2007). «Capacidad estatal, institucionalidad social y políticas públicas... o la búsqueda del "tesoro perdido" de la política social latinoamericana", en Alonso, G. (Ed.) Capacidades estatales, instituciones y política social, Buenos Aires, Prometeo. 
Rosas, A. y Gil, V. (2013). "La capacidad institucional de gobiernos locales en la atención del cambio climático. Un modelo de análogos", en Revista Mexicana de Análisis Político y Administración Pública, Departamento de Gestión Pública y Departamento de Estudios Políticos y de Gobierno Volumen II, número 2, julio-diciembre.

Rosas, A. (2008). «Una ruta metodológica para evaluar la capacidad institucional, en Política y Cultura, Núm. 30, DF, México, Universidad Autónoma Metropolitana-Xochimilco.

(2015a). La capacidad institucional de gobiernos locales para hacer frente al cambio climático. México, ITACA-Universidad Autónoma Metropolitana Unidad Xochimilco.

(2015b). Protocolo para el análisis de capacidades institucionales y de toma de decisiones referentes al cambio climático en el Instituto Nacional de Ecología y Cambio Climático y otras dependencias de gobierno federal. Informe final, Proyecto PNUD 86487.

Sartori, G. (2015). La política. Lógica y método en las ciencias sociales, Octava reimpresión, México, FCE.

Savitch, H.V. (1998). "Global challenge and institutional capacity: or, how we can refit local administration for the next century" en Administration \& Society, July 1998, vol. 30, nº 3.

Sikkink, K. (1993). "Las capacidades y la autonomía del Estado en Brasil y la Argentina. Un enfoque neoinstitucionalista", en Desarrollo Económico, vol. 32, n 138, Argentina, Instituto de Desarrollo Económico y Social.

Tilly, Ch. (1992). Coerción, capital y los Estados europeos, 990-1990, Madrid Alianza Editorial.

Tobelem, A. (1992). Institutional capacity analysis and development system (ICADS). Public Sector Management Division, Technical Department Latin America and the Caribbean Region of the World Bank. LATPS Occasional Paper Series No 9.

UNICEF (1999). Literature review: definitions of capacity building and implications for monitoring and evaluations, Draft Dec, Division of Evaluation, Policy and Planning, UNICEF New York.

Weiss, L. (1998). The myth of the powerless state, New York, Cornell University Press.

Willems, S. (2003). "Institutional Capacity and Climate Actions" en Environment Directorate International Energy Agency, nº 5, France, OECD.

Willems, S. y K. Baumert (2003). Institutional capacity and climate actions, France, OECD Environmental Directorate International Energy Agency.

\section{PARA CITAR ESTE ARTÍCULO:}

Rosas, A. (2019). "Capacidad institucional: revisión del concepto y ejes de análisis", DAAPGE, año 19, Nº 32 (ene-jun), 2019, pp. 81-107. Santa Fe, Argentina: UNL. 